\title{
Téoros
}

Revue de recherche en tourisme

\section{Sénégal : tourisme enclavé, tourisme intégré}

\section{Lailla Jabry, Diane Contant et Denise Tanguay}

Volume 5, numéro 1, mars 1986

Tourisme et régions

URI : https://id.erudit.org/iderudit/1080588ar

DOI : https://doi.org/10.7202/1080588ar

Aller au sommaire du numéro

Éditeur(s)

Université du Québec à Montréal

ISSN

0712-8657 (imprimé)

1923-2705 (numérique)

Découvrir la revue

Citer cet article

Jabry, L., Contant, D. \& Tanguay, D. (1986). Sénégal : tourisme enclavé, tourisme intégré. Téoros, 5(1), 16-17. https://doi.org/10.7202/1080588ar d'utilisation que vous pouvez consulter en ligne.

https://apropos.erudit.org/fr/usagers/politique-dutilisation/ 


\title{
Sénégal: tourisme enclavé, tourisme intégré
}

\author{
par Laila Jabry, Diane Contant et Denise Tanguay*
}

Depuis quelques années, le Sénégal constitue la destination privilégiée des "tours

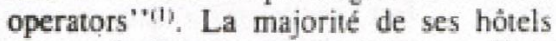
sont exclusivement réservés à cette clientèle. De novembre à mai, il est pratiquement impossible pour le touriste de passage de trouver à se loger.

Certains tours-operators proposent des circuits permettant de voir un maximum de choses en un minimum de temps. Le voyageur peut Également s'offrir un "circuitbrousse-intégré" "avec l'hébergement dans les villages. Il partagera alors la vie quotidienne des villageois.

Cet article se veut l'illustration des concepts suivants: le tourisme enclavé et le tourisme intégré traités respectivement à travers deux régions du Sénégal: le Cap Vert et la BasseCasamance. Notre texte n "est pas détaché de toute la problématique du tourisme international, c'est-à-dire la rencontre Nord/Sud. Aussi, commencerons-nous par rappeler le contexte d'apparition du tourisme au Sénégal.

Dans les années soixante, l'idée se répandit à l'intérieur des organisations d'aide internationale que le tourisme devait profiter au développement du tiers-monde en venant atténuer le déficit structurel de leur balance des paiements par l'apport de devises étrangères fortes.

Aujourd'hui, les flux touristiques que l'on promettait importants sont, malgré une croissance constante, toujours à l'avantage des pays industrialisés.

L'économie du.Sénégal basée sur l'exploitation de la cacahuète, des fruits de mer et sur le tourisme est assez dynamique comparativement à d'autres pays africains (revenus moyens per capita du Sénégal: 440 \$ U.S. contre 290 \$ U.S. pour la Gam= bie). Le Sénégal a pu investir énormément dans son industrie touristique; pourtant les gains retires seraient moindres que prèvus ${ }^{(2)}$. Le projet de développement d'un tourisme intégré, à travers la première expérience de Basse-Casamance, est la nou-

"Les auteures terminent leut baccalauréat en gestion et intervention touristiques a l'UDAM. Le present arti. cle est tire d'un travail pour le cours: espaces rouris. tiques (A, L. Sanguin) velle carte jouée pour retirer de meilleurs gains économiques.

\section{Les deux conceptions spatiales}

La forme dominante d'actions d'aménagement les plus visibles dans les pays en développement est celle qui se résume da l'exploitation de l'image du "pleasure resort' "exploitant les besoins dits des 5 "S" (sea, sand, sun, sex, scotch).

L'enclave est la surimposition d'une strucrure sur une autre. Elle peut être plus ou moins hermétique, représenter des barrières physiques (les clôtures du Club Méditerranéen) ou psychologiques (chapelet de complexes hôteliers) ou économiques (la "côte étoilée"" de la restauration). L'enclave se démarque de son environnement socio-économique par son băti et son style de vie. La région du Cap Vert témoigne du développement du tourisme enclavé.

La conception spatiale de l'intégration s'oriente de façon nouvelle vers des formes diffuses et kaléidoscopiques. Ces formes tendent vers une méme finalité, soit "la recherche $d$ 'une insertion harmonieuse dans le tissu social' ${ }^{\prime 3}$. Ceci peut s'exprimer à travers l'élargissement du territoire concerné, le regroupement des organismes prestataires des services d'hébergement. d'équipements de loisir et d'animation.

Le projet de Basse-Casamance de par son ampleur et sa cohérence interne se distingue des nombreuses tentatives en pays en développement. Il se présente comme une alternative en soi et non comme le simple prolongement exotique du séjour balnéaire.

\section{Cas du Sénégal}

A vol d'oiseau, le Sénégal apparaît comme une tété humaine au nez court et pointu, placee en figure de proue souriante à l'extrémité du bombement ouest africain. Son front baigne dans les eaux de l'Atlantique; sa chevelure borde les sables brûlants du désert de la Mauritanie; son cou est ancré dans le massif guinéen; son menton repose sur la forêt tropicale ${ }^{i 4}$.

Ce territoire de $196,192 \mathrm{~km}^{2}$ offre un échantillonage de tous les paysages, de tous les climats ainsi que de tous les peuples d'Afrique occidentale. Le Sénégal compte $6,700,000$ habitants. L'Islam, la religion officielle, est pratiquée par $86 \%$ de la population.

La ville de Dakar est bătie sur la moitié sud de la presqu'tile du Cap Vert. De novembre à mai, elle offre aux visiteurs un soleil assuré en méme temps que la fraicheur. Il

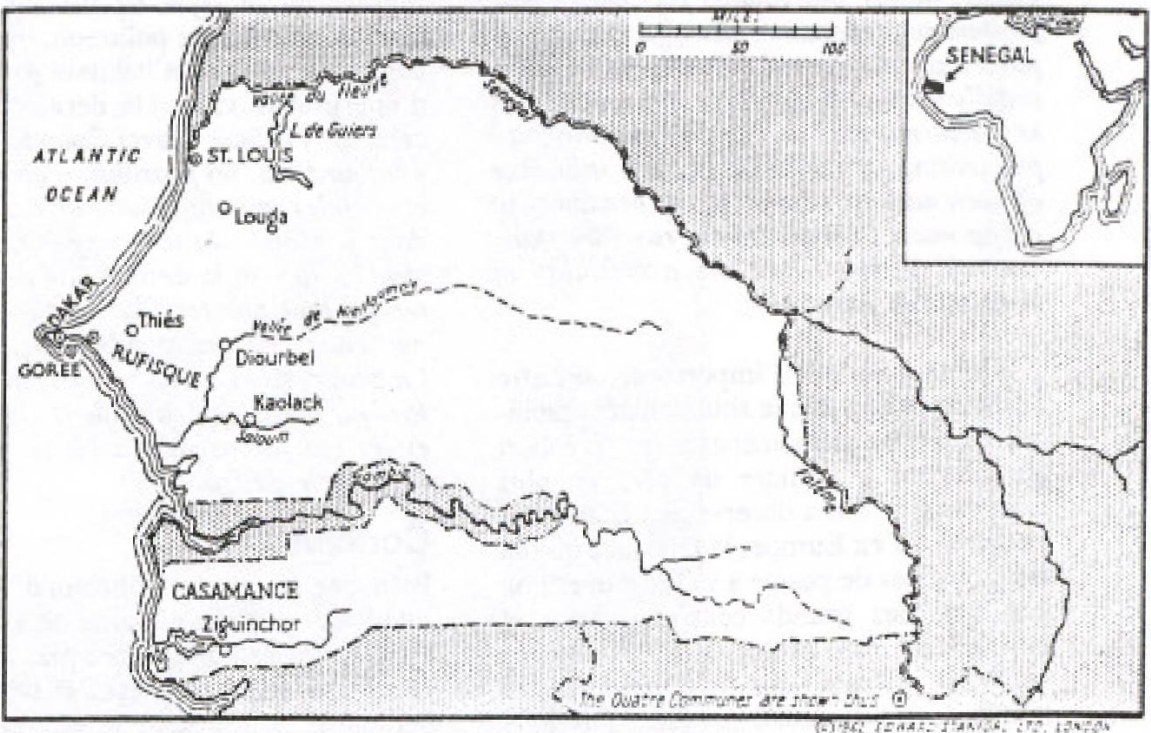


y a $1,300,000$ habitants à Dakar ${ }^{5}$. De l'époque coloniale, il reste peu de choses. Les villas et les hôtels particuliers ont disparu à cause de la spéculation foncière. Les tentatives de concilier les nécessités de l'architecture moderne avec son environnement africain ont été rares.

Quelques hôtels s'harmonisent avec le paysage. Leur décoration est de style africain. Un de ces hôtels est construit en forme de

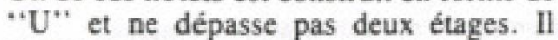
témoigne de la capacité de charge limitée des paysages.

L'offre d'hêbergement s'étale entre le palace de rêve, style super-production hollywoodienne, jusqu"au modeste hôtel à prix classé. La majorité des chaines hôtelières présentent un grand confort. Rien n'a été épargné pour le plaisir des amoureux du bronzage, du "farniente" et des sports. Saviez-vous que sans sortir de votre chambre, vous pourriez pécher de votre balcon surplombant la mer?

La restauration offre un choix qui s'étale entre la cuisine française ou sénégalaise, vietnamienne ou libanaise.

La Casamance, c'est avant tout un fleuve, long de $300 \mathrm{~km}$, qui se jette dans l'océan par un chenal principal et de multiples bras appelés "bolongs" . L'eau est salée et la marée, sensible. La variété de paysages, végétaux, hydrologiques, agricoles fait l'intérềt de la Basse-Casamance. Cette petite "Floride" sénégalaise est habitée par les plus anciennes populations du Sénégal, les Diolas et les Floups.

L'Agence de cooperation culturelle et technique (Agcop), née en 1971, est à l'origine du projet de la Basse-Casamance. Ce projet est à vocation plurinationale et réunit la participation du Sénégal, du Mali, du Niger et du Benin. Il a permis l'implantation de neuf campements qui disposent de 30 à 40 lots chacun.

La construction et l'aménagement des campements sont assurés par la population avec des matériaux locaux traditionnels (fondations en coquillages. murs en argile et chaux, nattes sur portes et fenettres, toits de chaume).

Ces campernents villageois présentent un confort minimal utilisant l'eau de puits, réfrigérateurs, douches, toilettes et cuisines collectives, mais sans électricité, ni climatisation ni véritable mobilier.

Les campements sont implantés tout au long du fleuve Casamance de manière à permettre un séjour agréable composé d'un grand nombre de petites excursions facilitant l'intégration avec la vie casamançaise sous toutes ses formes. Les touristes se déplacent en pirogue. mode de transport traditionnel.

Chaque campernent est animé et géré par la population du village sous forme de coo- pérative. Un bureau de 5 jeunes est ellu par I'assemblée générale du village pour assurer le fonctionnement quotidien du campement.

La vision du projet est de s'orienter vers un tourisme associatif et de partenariat dans lequel des relations étroites et durables pourraient se tisser entre l'équipe du campement et celle des voyageurs.

\section{Effets comparés du tourisme}

Au Cap Vert, les propriétés étrangères, telles que les multinationales, chaines hồtellières, transporteurs aériens, sont à la fois une source de revenu pour le Sénégal, par les taxes et impôts payes, et une fuite pour son économie nationale. Cette situation est agravée par l'endettement encouru lors de la construction des infrastructures (égouts, aéroports, etc.) et par l'importation massive de biens constituant I'offre standardisée (pellicules photographiques, vins, etc.).

Les emplois spécialisés sont encore souvent assurés par une main-d 'oeuvre étrangère tandis que les emplois non spécialisés et sous payés sont offerts aux nationaux. D'autres conséquences "classiques" sont 1'inflation et la spéculation foncière.

Enfin, certains diront que le tourisme enclavé entretient l'illusion du "paradis terrestre". II permet d'éviter un contact dérangeant et répond aux besoins de sécurité, d'hygiène, de confor physique et moral recherché par le vacancier des pays riches. Cependant, le principe de la ségrégation socio-spatiale peut relever d'un choix volontaire de la société hôte. Effectivement, il peut être un moyen de protection afin d'éviter la contagion de modèles de consommation incompatibles avec les ressources disponibles et le risque de heurter les valeurs traditionnelles.

Le projet de la Basse-Casamance, quant a lui, optimise les retombées économiques par la nationalisation des activités touristiques et l'utilisation stricte des seules ressources locales à l'intérieur d"un aménagement touristique intégré, ceci a toutes les étapes de la réalisation et de la gestion du projet. Les campements du projet sont un moyen efficace pour lutter contre l'exode rural. Ils permettent aux jeunes de trouver des emplois rémunérateurs.

"Le toral des investissements necessaires à la construction des 9 campements a atteint 22 millions de francs CFA, pour l'annee 1981, le chiffre d'affaires global s'est elevé à 23,807,000 de francs CFA et le bébéfice net atteint 12,440,000 francs CFA, "

Malgré le bilan économique positif de ce projet, les rapports entre visiteurs et visités soint loin d'être simples. Les valeurs traditionnelles se désagrègent sous le choc des idées modernes. La citation suivante en témoigne: "Les enfants tendent la main aux bords des campements pour mendier, alors qu 'ils ne l'ont jamais fait auparavant. ""?'.
Les initiateurs du projet affirment que parmi les formules touristiques existantes. le tourisme rural intégré se présente comme une des moins mauvaises. Une éducation à la connaissance plus intime de l'autre pourrait permettre des rapports plus respectueux de part et d'autre.

\section{L'avenir}

Des tendances pour l'avenir, en matière d'aménagement touristiques, peuvent-elle étre dégagées?

La Conférence internationale sur l'adaptation de la formation des personnels de tourisme et de voyage, tenue à Paris en juin 1985, mettait l'emphase sur des interventions globalistes. Les orientations qui seront favorisées prendront les formes suivantes:

"... créer des réseaux de senvice pour favoriser les mouvements de personnes des nationaux sur leur ternioire pour leurs fins variés (religion, sante, sciences, ans, commerce, etc.) et intégrer à ces réseaur Les étrangers venant pour des fins de vacances ou pour d'autres raisons... Si on pense d'abord aux besoins des nationaux et des régionaux, les services seront plus simples et respecterons la culture narionale; on y verra whe diversification d'we zone $a$ l'aulre... (4)

Cette position influente de ' $^{\prime} O$ MT $^{(9)}$ nous apparaît nettement en faveur d'un tourisme diffus qui se marierait harmonieusement à l'environnement-hôte sous toutes ses facettes: economiques, sociales, culturelles et de protection des paysages au sens large du terme. Les deux concepts d'aménagement touristiques présentent des avantages et des inconvénients. Le tourisme intégré parce qu'il permet aux nationaux d'ètre les maîtres d'oeuvres de leur développement et parce qu'il est un terrain propice à un véritable échange interpersonnel entre visiteurs et visités mérite sans doute d'être encouragé. $f$

\section{Notes at rifferences}

(11) VICTOH, Franco: Au Santgali, Paris, Hachette, quide visa, 1984

(2) KIRTLEY, Michel et Aubine: Senegambia: anow and fufure nation, National Geographic, vol. 168, no. 2, aoút 1985, pp. 224.251

(3) CAZES, Georges: Tourisme anelave, tourisme integré : le grand dabat de l'amenagement touriatiqu* dans las pars an developpomant. Ed. tions: Les Cahiers du Toutisme, Aix-men-Provence. 1983.

(4) Description inspirte du Guide bleu Hachatte. 1980, p. 31

(5) KIRTLEY, Michel en Aubine: op eif., p. 27

(6) CAP-IMPE. Anne-Marie: New Look pour le tou risme rural en Casamance. Jeune Afrique bconomie, no 33, 16 tevnier 1984

(7) idem, p. s8

(8) Extrait du compte rendu de mission de MM. M. Laplante et P. Bodson pour IUDAM

(9) L'OMT compte 105 duts-membres dont la moitie sont des pars en développement. 